\title{
Structural and functional alterations in heart and skeletal muscle following severe TAC in mice: impact of moderate exercise training
}

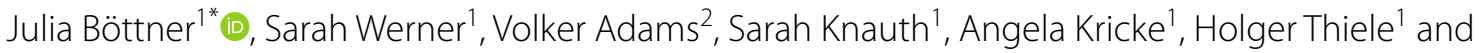

Petra Büttner ${ }^{1}$

\begin{abstract}
Background: Heart failure (HF) is the leading cause of death in western countries. Cardiac dysfunction is accompanied by skeletal alterations resulting in muscle weakness and fatigue. Exercise is an accepted interventional approach correcting cardiac and skeletal dysfunction, thereby improving mortality, re-hospitalization and quality of life. Animal models are used to characterize underpinning mechanisms. Transverse aortic constriction (TAC) results in cardiac pressure overload and finally HF. Whether exercise training improves cardiac remodeling and peripheral cachexia in the TAC mouse model was not analyzed yet. In this study, 2 weeks post TAC animals were randomized into two groups either performing a moderate exercise program (five times per week at $60 \% \mathrm{VO}_{2}$ max for 40 min for a total of 8 weeks) or staying sedentary.

Results: In both TAC groups HF characteristics reduced ejection fraction ( $-15 \%$ compared to sham, $\mathrm{p}<0.001)$, cardiac remodeling ( $+22.5 \%$ cardiomyocyte cross sectional area compared to sham; $p<0.001)$ and coronary artery congestion ( $+34 \%$ diameter compared to sham; $\mathrm{p}=0.008$ ) were observed. Unexpectedly, peripheral cachexia was not detected. Furthermore, compared to sedentary group animals from the exercise group showed aggravated HF symptoms [heart area $+9 \%(p=0.026)$, heart circumference $+7 \%(p=0.002)$, right ventricular wall thickness $-30 \%$ $(p=0.003)$ ] while muscle parameters were unchanged [Musculus soleus fiber diameter $(p=0.55)$, Musculus extensor digitorum longus contraction force $(p=0.90)$.
\end{abstract}

Conclusion: The severe TAC model is inappropriate to study moderate exercise effects in HF with respect to cardiac and skeletal muscle improvements. Further, the phenotype induced by different TAC procedures should be well documented and taken into account when planning experiments.

Keywords: Heart failure, Transverse aortic constriction, Moderate exercise, Intervention, Treadmill, Peripheral cachexia, Force production

\section{Introduction}

Approximately 64.3 million people worldwide suffer from heart failure (HF). In European countries the prevalence is up to $1.3-4 \%$ [1]. Despite continuously

*Correspondence: Julia.Boettner@medizin.uni-leipzig.de

${ }^{1}$ Department of Cardiology, Heart Center Leipzig at University of Leipzig, Strümpellstr. 39, 04289 Leipzig, Germany

Full list of author information is available at the end of the article improving therapies in HF the 5- and 10-year mortality still is estimated to be $53 \%$ and $65 \%$, respectively [2]. Two thirds of HF patients suffer from skeletal muscle atrophy [3], fatty infiltration, decreased antioxidant capacity and show muscle fiber type shift from slow-twitching to fast-twitching muscle fibers resulting in a metabolic shift from aerobic, oxidative to a rather anaerobic, glycolytic metabolism [4]. These molecular alterations in skeletal muscle structure and 
composition result in a loss of lean body mass, muscle weakness and fatigue [3]. Nevertheless, HF can be prevented or decelerated by modifying behavioral patterns. One easy-to-address and establish cardioprotective habit is physical activity, because it has the ability to correct the structural and metabolic alterations in the peripheral muscle [4-8]. Regular exercise is beneficial for cardiac health by reducing risk factors $[6$, 9]. Furthermore, exercise improves quality of life in HF patients by increasing exercise tolerance and oxygen uptake $[7,10,11]$. Exercise-based cardiac training is known to effectively reduce total and cardiovascular mortality as well as hospital admission [12].

Despite the accepted protective effect of exercise on HF, the molecular basis is still not completely understood. This is hindering the development of individualized exercise regimens and the identification of underpinning regulatory pathways that may be targeted pharmaceutically.

Different animal models, that imitate human pathologies, are accessible for HF induction. In patients with HF due to myocardial infarction exercise improves cardiac remodeling and function [13]. Thus, ligation of the left anterior descending artery (LAD) is a common method to induce myocardial infarction and subsequently HF in animal models. A drawback of this model is high mortality and low reproducibility [14].

To overcome these obstacles transverse aortic constriction (TAC) is used to induce HF whereas low mortality and high reproducibility are reported for this method [15]. TAC limits the left ventricular (LV) outflow resulting in LV pressure overload and fast evolving HF characterized by cardiac hypertrophy and remodeling as well as decreasing cardiovascular performance $[16,17]$. To standardize the procedure a needle is placed next to the aorta, a full constriction is placed and after the needle is removed, the final aortic diameter is determined. Lower needle diameters determine more severe hypertrophy as well as accelerated time course of HF development [15, 16]. Mild TAC introduced by aortic banding using a 25 gauge (G) needle represents a mild model of hypertrophy and diastolic dysfunction without systolic dysfunction or cardiac fibrosis, consistent with the human condition of hypertensive heart disease [16]. Moderate 26G TAC results in systolic dysfunction with perivascular cardiac fibrosis.
Severe TAC applied by using a $27 \mathrm{G}$ needle eventuates in systolic and diastolic dysfunction, distinct cardiac fibrosis, hypertrophy and secondary increased lung mass, consistent with pulmonary edema [16].

The effect of exercise on HF development and associated muscle remodeling using the TAC model in mice was studied by Wang et al. and Tian et al. $[8,18]$. They observed improved cardiac performance following exercise training with increased intensity [8]. Most existing studies on TAC in combination with exercise intervention focus on cardiac effects and lack information about TAC- and exercise-induced structural, functional and metabolic alterations of the skeletal muscles. On that account, we applied severe cardiac pressure overload by banding the aorta using a $27 \mathrm{G}$ needle to analyze the effects of moderate training in HF on cardiac and skeletal muscle structure and function. To our best knowledge, this is the first study to address both, cardiac and skeletal muscle performance in HF mice after exercise intervention.

\section{Results \\ Evaluation of TAC phenotype}

In the $48 \mathrm{~h}$ post-operative period six mice died following $27 \mathrm{G}$ severe TAC, two mice died following sham surgery. In $\mathrm{TAC}_{\mathrm{SED}}$ one mouse died $22 \mathrm{~d}$ post-surgery from unknown cause, which led to final groups of $n=13$ in sham, $\mathrm{n}=11$ in sedentary $\left(\mathrm{TAC}_{\mathrm{SED}}\right)$ and $\mathrm{n}=12$ in exercising TAC $\left(\mathrm{TAC}_{\mathrm{EX}}\right)$ groups.

Echocardiographic measurements (Fig. 1A) confirmed establishment of TAC 2 weeks after surgery. The velocity in the left common carotid artery (LCCA) decreased after TAC $(\mathrm{p}<0.001)$, whereas the right common carotid artery (RCCA) velocity only numerically increased after TAC compared to sham-operated animals $(\mathrm{p}=0.098)$.

Ten weeks post-surgery body weights (BW) and tibial length (TL) in $\mathrm{TAC}_{\mathrm{SED}}$ and $\mathrm{TAC}_{\mathrm{EX}}$ were comparable to sham. All results are summarized in Table 1.

\section{Severe TAC alters cardiac structure and function in sedentary animals}

Cardiac remodeling after TAC operation was evaluated by analysis of heart metrics, development of interstitial and perivascular fibrosis as well as coronary artery remodeling 10 weeks post TAC (see Fig. 2 and Table 1).

(See figure on next page.)

Fig. 1 Cardiac characteristics 1 week before TAC surgery (-1), 2 weeks post TAC surgery (2), and 10 weeks post TAC whereas TAC EX $_{\text {animals }}$ did 8 weeks of moderate training. Quantification of the left (LCCA, left graph) and right common carotid (RCCA, right graph) velocity (A), ejection fraction (B) and left ventricular (LV) volume (C) and posterior wall thickness (D) during diastolic (left) and systolic (right) phase using echocardiography. Mean \pm SEM. $n \geq 11$. Two-way ANOVA with Bonferroni correction. ${ }^{*} p<0.05,{ }^{* *} p<0.01,{ }^{* * *} p<0.001$, vs. sham 

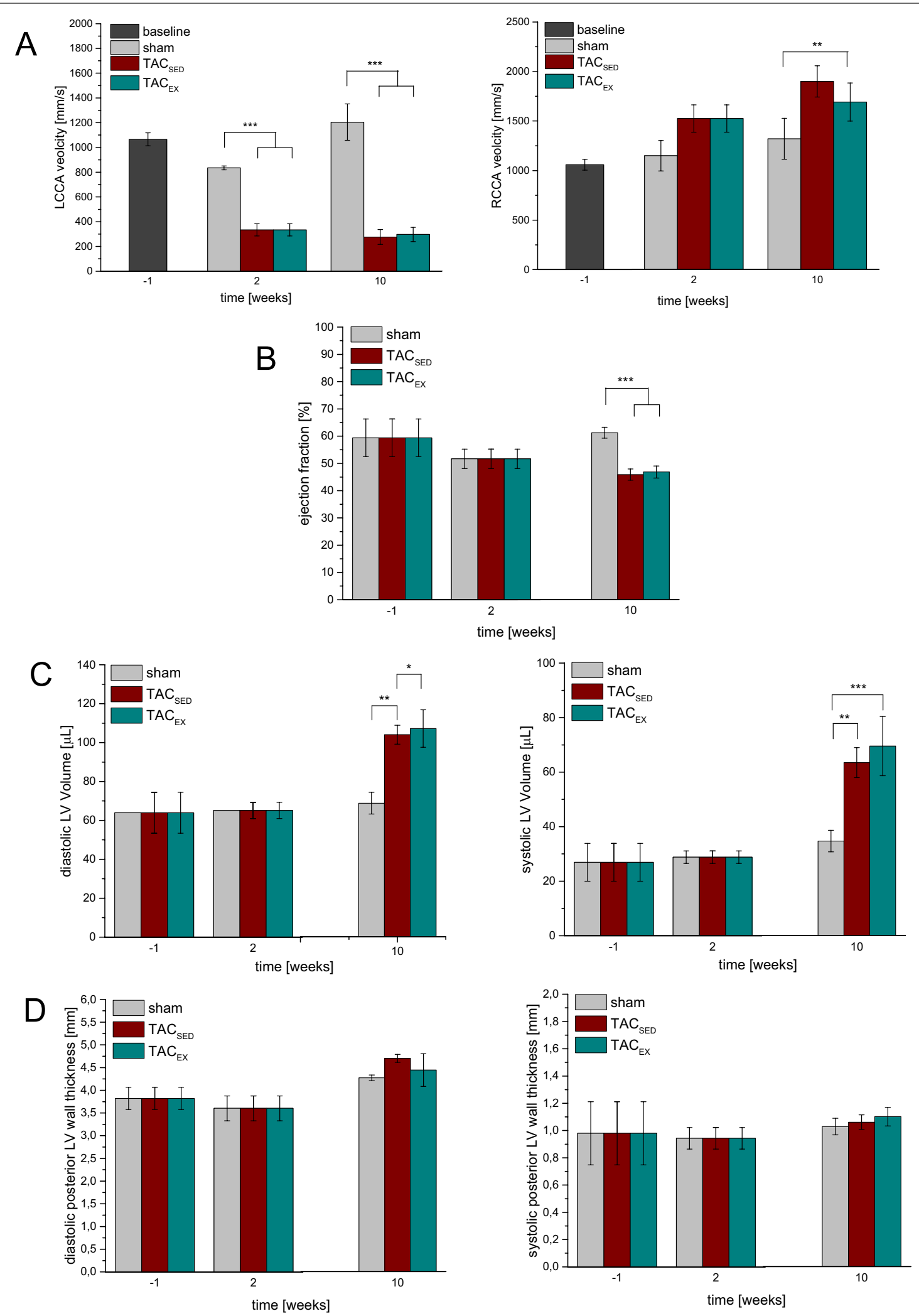

Fig. 1 (See legend on previous page.) 
Table 1 Functional and morphological characteristics of sham, $T A C_{S E D}$ and $T A C_{E X}$

\begin{tabular}{|c|c|c|c|}
\hline & Sham & $\mathrm{TAC}_{\mathrm{SED}}$ & $\mathrm{TAC}_{\mathrm{EX}}$ \\
\hline \multicolumn{4}{|l|}{ Baseline TAC phenotype } \\
\hline Body weight (g) & $28.2 \pm 0.1$ & $28.2 \pm 0.2(p=0.13)$ & $29.2 \pm 0.1(\mathrm{a}: p=0.95 ; \mathrm{b}: p=0.18)$ \\
\hline Tibia length (mm) & $17.2 \pm 0.05$ & $17.5 \pm 0.03(p=0.89)$ & $17.2 \pm 0.05(\mathrm{a}: p=0.10 ; \mathrm{b}: p=0.12)$ \\
\hline Heart weight (mg) & $145.6 \pm 1.0$ & $201.4 \pm 2.8(p<0.001)$ & $213.8 \pm 2.8(\mathrm{a}: p<0.001 ; \mathrm{b}: p=0.46)$ \\
\hline Heart weight/tibia length (mg/mm) & $8.5 \pm 0.1$ & $11.6 \pm 0.1(p<0.001)$ & $12.4 \pm 0.2(\mathrm{a}: p<0.001 ; \mathrm{b}: p=0.26)$ \\
\hline $\mathrm{EDL}$ mass/TL (mg/mm) & $0.77 \pm 0.12$ & $0.83 \pm 0.05(p=0.14)$ & $0.81 \pm 0.05(\mathrm{a}: p=0.35 ; \mathrm{b}: p=0.61)$ \\
\hline \multicolumn{4}{|l|}{ Functional cardiac parameters } \\
\hline $\begin{array}{l}\text { LCCA velocity } 2 \text { weeks post TAC } \\
(\mathrm{mm} / \mathrm{s})\end{array}$ & $833.5 \pm 15.3$ & \multicolumn{2}{|c|}{$333.0 \pm 49.5(\mathrm{a}: p<0.001 ; \mathrm{b}: p=0.90)$} \\
\hline $\begin{array}{l}\text { RCCA velocity } 2 \text { weeks post TAC } \\
(\mathrm{mm} / \mathrm{s})\end{array}$ & $1148.7 \pm 154.4$ & \multicolumn{2}{|c|}{$1524.1 \pm 138.0(\mathrm{a}: p=0.098 ; \mathrm{b}: p=0.42)$} \\
\hline LVEF (\%) & $61 \pm 5$ & $46 \pm 6(p<0.001)$ & $47 \pm 7(\mathrm{a}: p<0.001 ; \mathrm{b}: p=0.76)$ \\
\hline FS (\%) & $32 \pm 3$ & $22 \pm 4(p<0.001)$ & $23 \pm 4(\mathrm{a}: p<0.001 ; \mathrm{b}: p=0.76)$ \\
\hline Diastolic LV volume $(\mu \mathrm{L})$ & $68.7 \pm 5.58$ & $103.9 \pm 4.9(p=0.002)$ & $107.1 \pm 9.6(\mathrm{a}: p=0.03 ; \mathrm{b}: p=0.77)$ \\
\hline Systolic LV volume $(\mu \mathrm{L})$ & $34.6 \pm 4.0$ & $63.4 \pm 5.5(p<0.001)$ & $69.4 \pm 10.9(\mathrm{a}: p=0.007 ; \mathrm{b}: p=0.63)$ \\
\hline Diastolic LV wall thickness (mm) & $4.3 \pm 0.1$ & $4.7 \pm 0.9(p=0.27)$ & $4.4 \pm 0.4(a: p=0.097 ; b: p=0.69)$ \\
\hline Systolic LV wall thickness (mm) & $1.0 \pm 0.1$ & $1.1 \pm 0.1(p=0.64)$ & $1.1 \pm 0.1(a: p=0.50 ; b: p=0.82)$ \\
\hline \multicolumn{4}{|c|}{ Histological evaluation of cardiac remodeling } \\
\hline Relative heart area & 1 & $0.98 \pm 0.05(p=0.72)$ & $1.09 \pm 0.03(\mathrm{a}: p=0.022 ; \mathrm{b}: p=0.053)$ \\
\hline Relative circumference & 1 & $0.98 \pm 0.02(p=0.45)$ & $1.07 \pm 0.02(\mathrm{a}: p=0.01 ; \mathrm{b}: p=0.003)$ \\
\hline Relative LV thickness & 1 & $1.09 \pm 0.08(p=0.40)$ & $1.09 \pm 0.06(a: p=0.23 ; b: p=0.97)$ \\
\hline Relative RV thickness & 1 & $0.77 \pm 0.08(p=0.044)$ & $0.70 \pm 0.06(\mathrm{a}: p=0.004 ; \mathrm{b}: p=0.56)$ \\
\hline Cardiomyocyte CSA $\left(\mu m^{2}\right)$ & $50.3 \pm 0.8$ & $61.6 \pm 1.2(p<0.001)$ & $59.6 \pm 1.0(a: p<0.001 ; b: p=0.20)$ \\
\hline Perivascular fibrosis (\%) & $1.8 \pm 0.1$ & $2.0 \pm 1.1(p=0.75)$ & $2.6 \pm 0.2(a: p=0.27 ; b: p=0.48)$ \\
\hline Interstitial fibrosis (\%) & $0.2 \pm 0.1$ & $0.4 \pm 0.1(p=0.51)$ & $0.3 \pm 0.1(a: p=0.01 ; b: p=0.86)$ \\
\hline Coronary artery diameter $(\mu \mathrm{m})$ & $27.3 \pm 2.0$ & $36.6 \pm 2.8(p=0.008)$ & $30.8 \pm 3.0(\mathrm{a}: p=0.32 ; \mathrm{b}: p=0.16)$ \\
\hline \multicolumn{4}{|l|}{ Muscle function and remodeling } \\
\hline EDL max. force $\left(\mathrm{N} / \mathrm{cm}^{2}\right)$ & $25.8 \pm 5.7$ & $28.8 \pm 4.7(p=0.20)$ & $28.1 \pm 6.6(\mathrm{a}: p=0.30 ; \mathrm{b}: p=0.9)$ \\
\hline EDL fiber diameter $(\mu \mathrm{m})$ & $40.7 \pm 1.7$ & $35.3 \pm 1.6(p=0.04)$ & $37.1 \pm 1.4(\mathrm{a}: p=0.16, \mathrm{~b}: p=0.79)$ \\
\hline EDL slow fiber proportion (\%) & $2.25 \pm 0.41$ & $1.81 \pm 0.48(p=0.78)$ & $2.63 \pm 1.12(\mathrm{a}: p=0.62 ; \mathrm{b}: p=0.53)$ \\
\hline EDL fast fiber proportion (\%) & $92.7 \pm 1.1$ & $86.6 \pm 07.2(p=0.32)$ & $93.1 \pm 4.6(\mathrm{a}: p=0.84 ; \mathrm{b}: p=021)$ \\
\hline Soleus fiber diameter ( $\mu \mathrm{m})$ & $44.3 \pm 1.9$ & $45.8 \pm 1.5(p=0.74)$ & $37.8 \pm 1.5(\mathrm{a}: p=0.01 ; \mathrm{b}: p=0.002)$ \\
\hline Soleus slow fiber proportion (\%) & $30.3 \pm 3.0$ & $33.6 \pm 8.5(p=0.73)$ & $29.7 \pm 2.6(\mathrm{a}: p=0.87 ; \mathrm{b}: p=0.78)$ \\
\hline
\end{tabular}

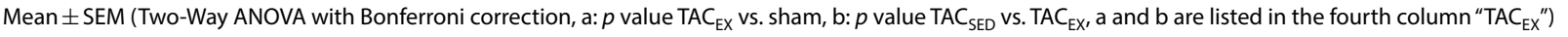
$E D L$ extensor digitorum longus, $L V E F$ left ventricular ejection fraction, $F S$ fractional shortening, $R V$ right ventricle, CSA cross-sectional area

The area and circumference in $\mathrm{TAC}_{\mathrm{SED}}$ were comparable to the sham operated control group. LV thickness of heart cross sections was slightly decreased in $\mathrm{TAC}_{\mathrm{SED}}$. Interestingly, the right ventricular (RV) wall thickness in $\mathrm{TAC}_{\mathrm{SED}}$ was significantly reduced by $22 \%$ in comparison to the sham group $(\mathrm{p}=0.049$ see Fig. 2A). Cardiomyocyte cross sectional area (CSA) in $\mathrm{TAC}_{\mathrm{SED}}$ was significantly increased by $22 \%$ in contrast to sham (see Fig. 2B). The amount of interstitial and perivascular fibrosis in $\mathrm{TAC}_{\mathrm{SED}}$ was comparable to sham (see Fig. 2D). The coronary artery diameter in $\mathrm{TAC}_{\mathrm{SED}}$ was significantly increased by $9 \mu \mathrm{m}(\mathrm{p}=0.008$ vs. sham; Fig. $2 \mathrm{E}$ ). The heart weight of the $\mathrm{TAC}_{\mathrm{SED}}$ was increased up to 1.4-fold in contrast to sham. Echocardiographic analyses revealed an increase in diastolic LV volume by $50 \%(\mathrm{p}=0.002)$, but no significant alterations in $\mathrm{LV}$ posterior wall thickness $(\mathrm{p}=0.27)$ as well as a significant reduction in left ventricular ejection fraction (LVEF) of $15 \%$ compared to sham in $\mathrm{TAC}_{\mathrm{SED}}$. Additionally, systolic LV volume was significantly increased by $28 \mu \mathrm{L}(\mathrm{p}<0.001)$, although LV wall thickness in the systolic phase was unaltered in $\operatorname{TAC}_{\mathrm{SED}}(\mathrm{p}=0.64)$. 


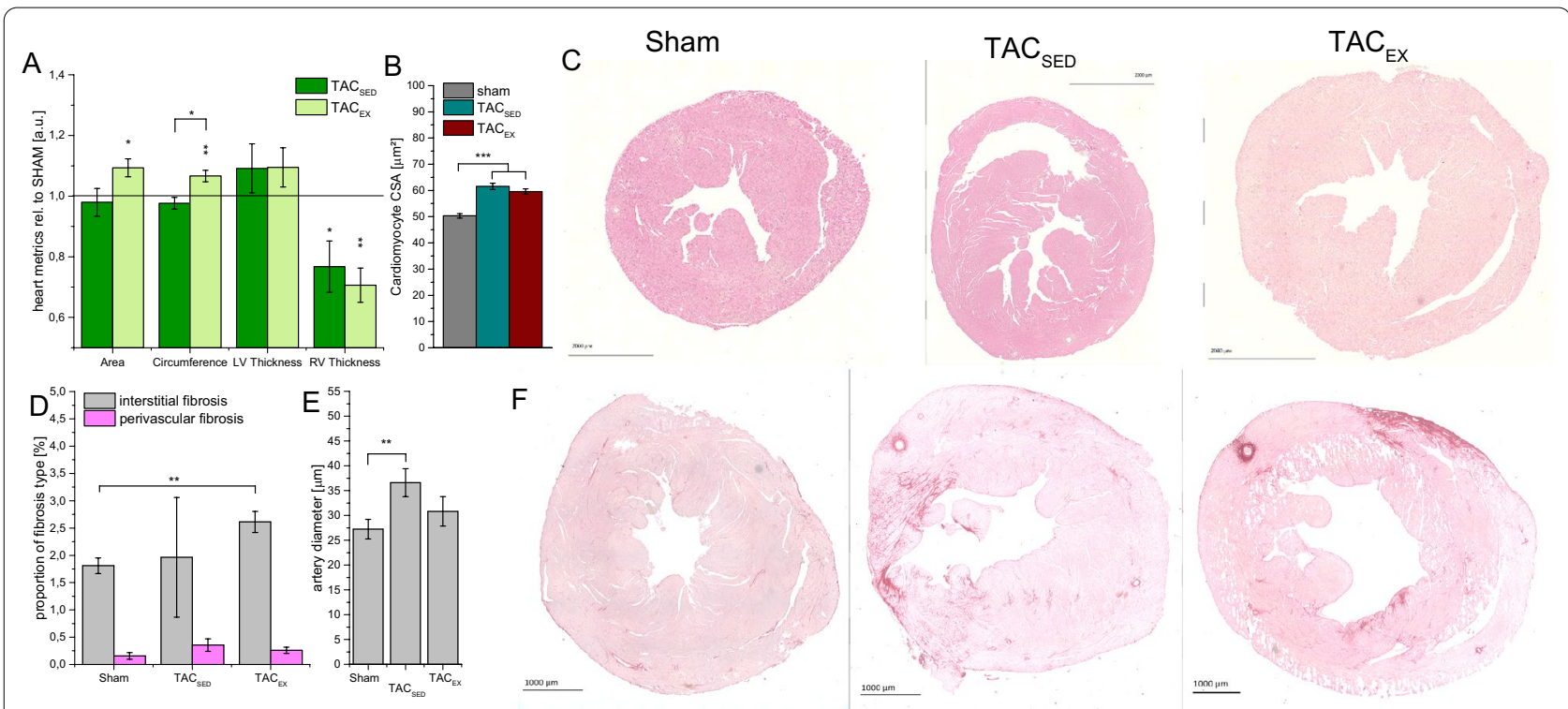

Fig. 2 Effects of pressure overload and moderate training on cardiac hypertrophy and fibrosis. A Calculated area, circumference, left ventricular (LV) and right ventricular (RV) wall thickness of TAC-operated mice in relation to sham-operated control, B cardiomyocyte cross-sectional area (CSA), C hematoxylin/eosin stained heart cross-sections. D Quantification of the TAC-induced fibrosis and $\mathbf{E}$ coronary artery diameter using $\mathbf{F}$ Sirius-Red stained heart cross-sections. Mean $\pm \mathrm{SEM}, n \geq 5$. One-way ANOVA with Bonferroni correction. ${ }^{*} p<0.05,{ }^{* *} p<0.01,{ }^{* * *} p<0.001$

\section{Exercise effects on TAC induced cardiac dysfunction}

We analyzed the impact of moderate exercise training $\left(\mathrm{TAC}_{\mathrm{EX}}\right)$ on the cardiac alterations observed in $\mathrm{TAC}_{\mathrm{SED}}$ (Fig. 2 and Table 1). Cardiac CSA was increased by $8 \%$ $(\mathrm{p}=0.026)$ and heart circumference was increased by $6 \%(\mathrm{p}=0.002)$, while RV wall thickness was decreased by $30 \%(p=0.003)$ in relation to sham. RV wall thickness reduction was observed in $\mathrm{TAC}_{\mathrm{SED}}(23 \%)$ and TAC EX (24\%) without differences between these two groups $(\mathrm{p}=0.56)$. In $\mathrm{TAC}_{\mathrm{EX}}$ cardiomyocyte CSA was increased by $18 \%$ in contrast to sham $(\mathrm{p}<0.001)$. In comparison to $\mathrm{TAC}_{\mathrm{SED}}$, cardiomyocyte CSA remained similar in $\mathrm{TAC}_{\mathrm{EX}}(\mathrm{p}=0.56)$. $\mathrm{TAC}_{\mathrm{EX}}$ showed a 1.44-fold rise in cardiac interstitial fibrosis compared to sham $(p=0.01)$. No difference in the amount of interstitial fibrosis in $\mathrm{TAC}_{\mathrm{SED}}$ and $\mathrm{TAC}_{\mathrm{EX}}$ was observed. The amount of perivascular fibrosis in $\mathrm{TAC}_{\mathrm{EX}}$ was comparable to sham and to $\mathrm{TAC}_{\mathrm{SED}}$. Numerically, exercise intervention in the TAC operated group reduced the coronary artery diameter in the myocardium by $6 \mu \mathrm{m}$ in $\mathrm{TAC}_{\mathrm{EX}}$ compared to $\mathrm{TAC}_{\mathrm{SED}}$. Heart weight in $\mathrm{TAC}_{\mathrm{EX}}$ was significantly increased in contrast to sham $(\mathrm{p}<0.001)$. No difference in heart weight between $\mathrm{TAC}_{\mathrm{SED}}$ and TAC EX was found. Functional cardiac parameters of $\mathrm{TAC}_{\mathrm{EX}}$ are shown in Fig. 1. Diastolic and systolic LV volume in $\mathrm{TAC}_{\mathrm{SED}}$ and in $\mathrm{TAC}_{\mathrm{EX}}$ remained comparable. The diastolic and systolic LV wall thickness in TAC $_{\text {SED }}$ were not improved in $\mathrm{TAC}_{\mathrm{EX}}$. LV volume in diastolic and systolic phase remained 1.5- and twofold increased and the LVEF was reduced by $24 \%(\mathrm{p}<0.001)$ compared to sham. Additionally, no differences of LV output were found in $\mathrm{TAC}_{\mathrm{SED}}$ and in $\mathrm{TAC}_{\mathrm{EX}}$.

\section{Functional and structural alterations in skeletal muscle in sedentary and trained TAC mice}

Effects of moderate exercise on HF were analyzed focusing on cardiac characteristics as well as peripheral muscle alterations. Extensor digitorum longus (EDL) contraction force, muscle fiber diameter and the proportion of slow-twitch and fast-twitch fibers in the EDL and the soleus muscle were analyzed to study the effects of moderate exercise in TAC induced HF 10 weeks after TAC surgery (see Table 1 and Fig. 3 ).

EDL contraction force was significantly increased in $\mathrm{TAC}_{\mathrm{SED}}$ compared to sham $(\mathrm{p}=0.017)$, but not in $\mathrm{TAC}_{\mathrm{EX}}$. The EDL fiber diameter was significantly lower in $\mathrm{TAC}_{\mathrm{SED}}(\mathrm{p}=0.044)$ but not in $\mathrm{TAC}_{\mathrm{EX}}(\mathrm{p}=0.16)$, whereas there was no difference between both TAC groups.

Soleus muscle fiber diameter in $\mathrm{TAC}_{\mathrm{SED}}$ remained comparable to sham. Interestingly, soleus muscle fiber diameter was significantly decreased $(p=0.002)$ in TAC EX compared to sham. No alterations in the amount of slow and fast muscle fibers in EDL as well as in slow muscle fibers in soleus were observed. 


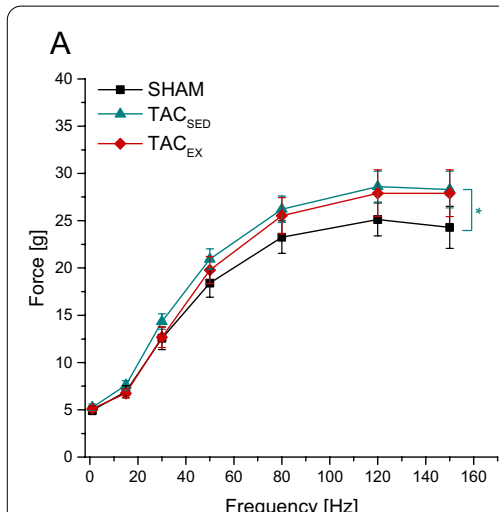

D Sham

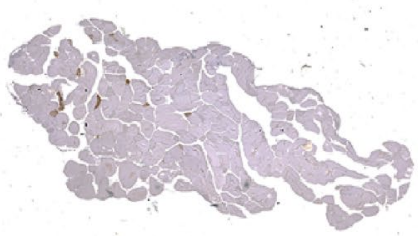

$500 \mu \mathrm{m}$

E

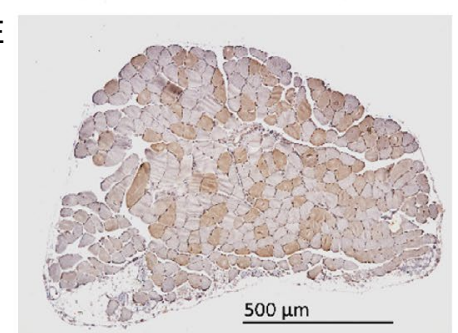

B

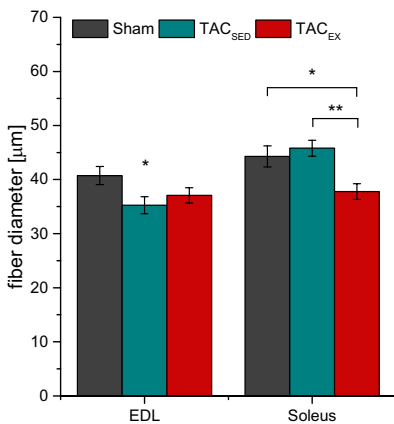

EDL

TAC $_{\text {SED }}$

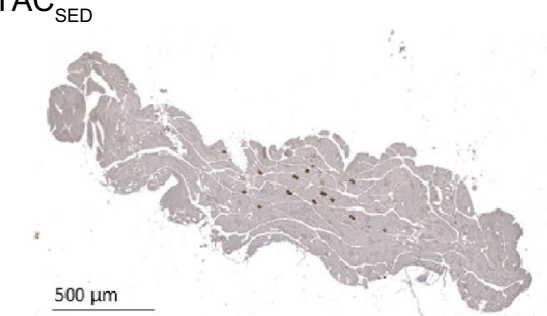

$500 \mu \mathrm{m}$

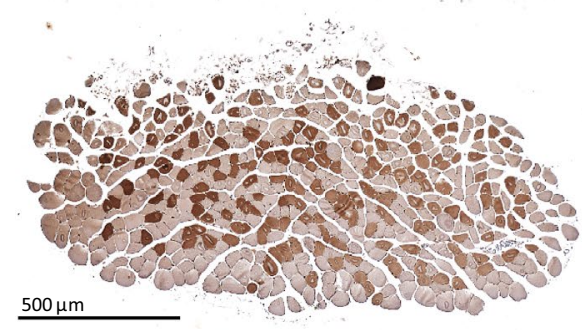

C
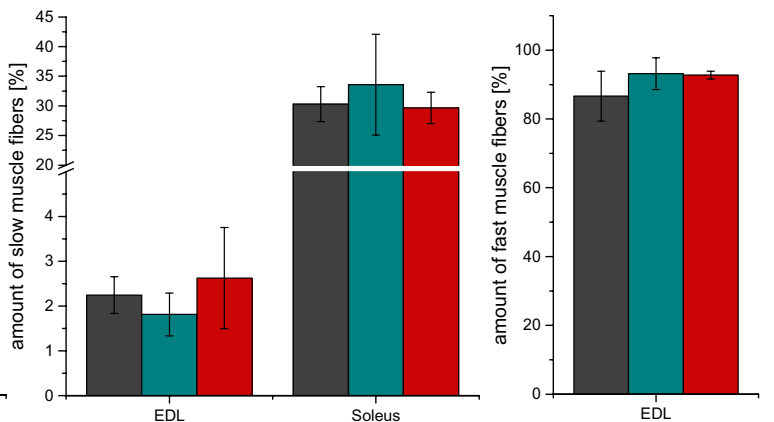

EDL

$\mathrm{TAC}_{\mathrm{EX}}$

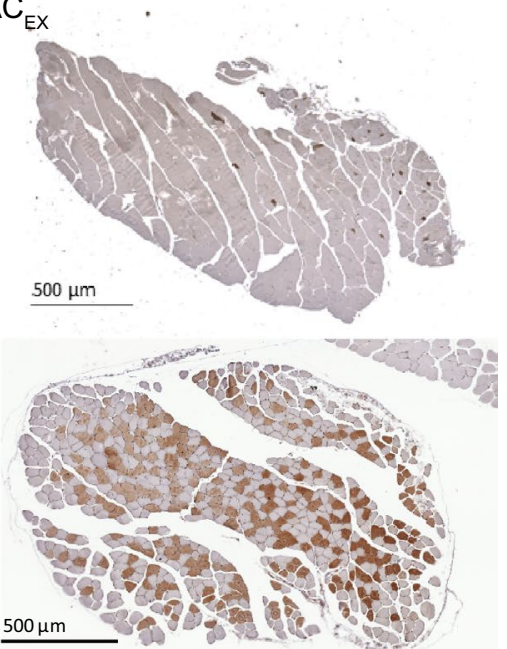

Fig. 3 Effects of moderate training on muscle function of fast-twitching Extensor digitorum longus (EDL) and slow-twitching Soleus 10 weeks after TAC. In vitro absolute muscle forces of EDL (A) and fiber diameter (B). Proportion of slow and fast fibers (C) and representative images of slow muscle fiber staining in EDL (D) and Soleus (E). Mean \pm SEM. Two-Way ANOVA with Bonferroni's correction. ${ }^{*} p<0.05,{ }^{* *} p<0.01,{ }^{* * *} p<0.001$

\section{Discussion}

We applied 27G severe TAC to induce a severe HF phenotype but chose a moderate exercise regimen to avoid overcharging TAC mice by exercise. Resulting cardiac dysfunction and peripheral cachexia as well as the potential capability of moderate exercise to improve them was examined. The results of the study can be summarized as follows:

1. additional aggravation of cardiac remodeling after exercise intervention

2. similar cardiac performance independently from exercise intervention

3. comparable structure and function of skeletal muscles in both, $\mathrm{TAC}_{\mathrm{SED}}$ and $\mathrm{TAC}_{\mathrm{EX}}$

4. EDL cross-sectional area was improved by exercise intervention, whereas the Soleus muscle showed further fiber atrophy following exercise intervention

\section{LV remodeling and dysfunction in severe TAC}

In the present study $27 \mathrm{G}$ severe TAC induced HF characteristics in the mouse model. Increased diastolic and systolic LV filling accompanied by a significantly reduced EF were found, indicating LV dilatation and dysfunction as hallmarks of HF. The observed interstitial fibrotic and coronary artery remodeling is also in accordance to other studies [16, 19]. Cardiac remodeling in the TAC mouse model is accompanied by a congestion into coronary vessels [19] resulting in an increase in coronary artery diameter. We found an 1.34-fold increased coronary artery diameter in TAC SED in comparison to sham, whereas Yang et al. found coronary artery diameter to be further increased by 2.3-fold [19].

In the current study no increase in perivascular fibrosis was observed while Richards et al. found increased perivascular fibrosis 4 weeks post $26 \mathrm{G}$ moderate TAC. Noteworthy, Richards et al. observed no interstitial 
fibrosis [16]. Yang et al. found interstitial and perivascular fibrosis 5 weeks after TAC in male mice (no needle diameter specified) [19]. Wang et al. [8] reported significant fibrosis 10 weeks after TAC (no needle diameter specified, presumably 27G [20]). Taken together, the development and pattern of fibrosis seems to depend strongly on the used needle diameter and the progressed time post TAC $[7,8]$. Notably, our data suggest that the development of fibrosis in $\mathrm{TAC}_{\mathrm{SED}}$ is highly individual, which is contrary to the concept that TAC results in an uniform and reproducible development of HF [15].

Based on the current results, future studies should include the specific TAC phenotype in their hypothesis generation including a detailed description of the performed TAC procedure as well as mouse strain, sex, age and origin. Furthermore, the high inter-individual variability in cardiac remodeling observed in this study should be taken into account when planning TAC experiments with regards to animal numbers and sample size calculation.

\section{$\mathrm{RV}$ remodeling in severe pressure overload}

While we found no differences in LV wall thickness following TAC surgery, the RV wall was significantly thinner in both TAC groups. The echocardiographic analysis of the lateral RV wall thickness confirmed diastolic RV dilation, maybe resulting in RV dysfunction. $\mathrm{RV}$ dilatation as a consequence of LV pressure overload was addressed in only a few studies [21, 22] and thereby remains elusive. In pulmonary hypertension (PH) RV dilatation was found to represent an adjustment mechanism to reduce RV load [23]. Our data are in line with recent findings in TAC operated mice that showed distinct RV remodeling and diastolic dysfunction [22]. Platt et al. applied 26G TAC and monitored structural and functional RV and LV remodeling over 18 weeks. They postulated diastolic RV dysfunction to occur secondary to LV dysfunction. Finally, they showed establishment of PH secondary to RV dysfunction [22]. Interestingly, Platt et al. [22] found RV and LV peak pressure 4 weeks post TAC. While LV pressure normalized thereafter RV pressure remained elevated. This is well in line with our observations 10 weeks post TAC.

Right heart failure due to chronic RV overload is the major cause of death among patients with $\mathrm{PH}$ [24]. PH is associated with high mortality and its treatment is still challenging [25]. Furthermore, there is an unfortunate lack of sufficient animal models for PH. Therefore, we suggest the characterization of the TAC mouse model as a suitable model for PH type 2 [22], which is secondary to left heart disease [26].

\section{Result classification in the context of TAC induced HF and exercise intervention in mice}

Numerous clinical and experimental studies showed that exercise improves cardiac structure and performance in ischemic cardiomyopathy [27-29], in systemic hypertension models [30, 31], in myocardial infarction [7] and reduces the rate of re-infarction and mortality in patients with HF [5]. Exercise-induced cardioprotection is underpinned by various molecular mechanisms, such as activation of heat shock and ER stress proteins as well as cyclooxygenase, enhancing the antioxidant capacity and autophagy processes [32]. Several studies investigated cardiac and skeletal muscle performance using mild to severe TAC with or without exercise in mice $[8,18]$. Studies on TAC and exercise intervention always applied exercise as secondary intervention. Independently of the different starting times for exercise intervention ( $0-14$ days post TAC) in the different studies, TAC was established at any time post-surgery, but no HF characteristics were verifiable at the exercise starting time.

Nine weeks of swimming exercise with an increasing duration were reported to induce beneficial cardiac effects in severe TAC operated mice [8]. LV mass was lower in comparison to $\mathrm{TAC}_{\mathrm{SED}}$ while $\mathrm{EF}$ and FS were improved, indicating at least a partial recovery of cardiac output. Further, cardiomyocyte CSA, amount of fibrosis, catecholamines, nitric oxide and reactive oxygen species levels were improved in this study [8]. Correspondingly, improved LV mass, EF, FS, cardiomyocyte CSA, atrial and brain natriuretic peptide levels were reported following 8 weeks of treadmill running with increasing intensity in mice following severe TAC [18]. Due to these studies, we expected an improvement of HF-induced cardiac and skeletal muscle function following exercise training.

Instead, we observed more pronounced cardiac hypertrophy and thinning of the RV wall in TAC EX. Diastolic as well as systolic LV volume and wall thickness in $\mathrm{TAC}_{\mathrm{EX}}$ were similar to $\mathrm{TAC}_{\mathrm{SED}}$, indicating no beneficial effects of moderate exercise on cardiac remodeling or output. Exclusively, coronary artery remodeling induced by pressure overload may be at least partially reversed or prevented by exercise training.

Therefore, we comprehensively compared our data with the current literature on TAC-induced HF with a focus on exercise intervention or muscle function (Table 2). Van Deel et al. combined 25G mild TAC with 8 weeks of voluntary wheel run [6], which is better classified as a mild exercise training regime. Importantly, the TAC mice reduced their voluntary run distance indicating decreased wellbeing as observed in $\mathrm{HF}$ 


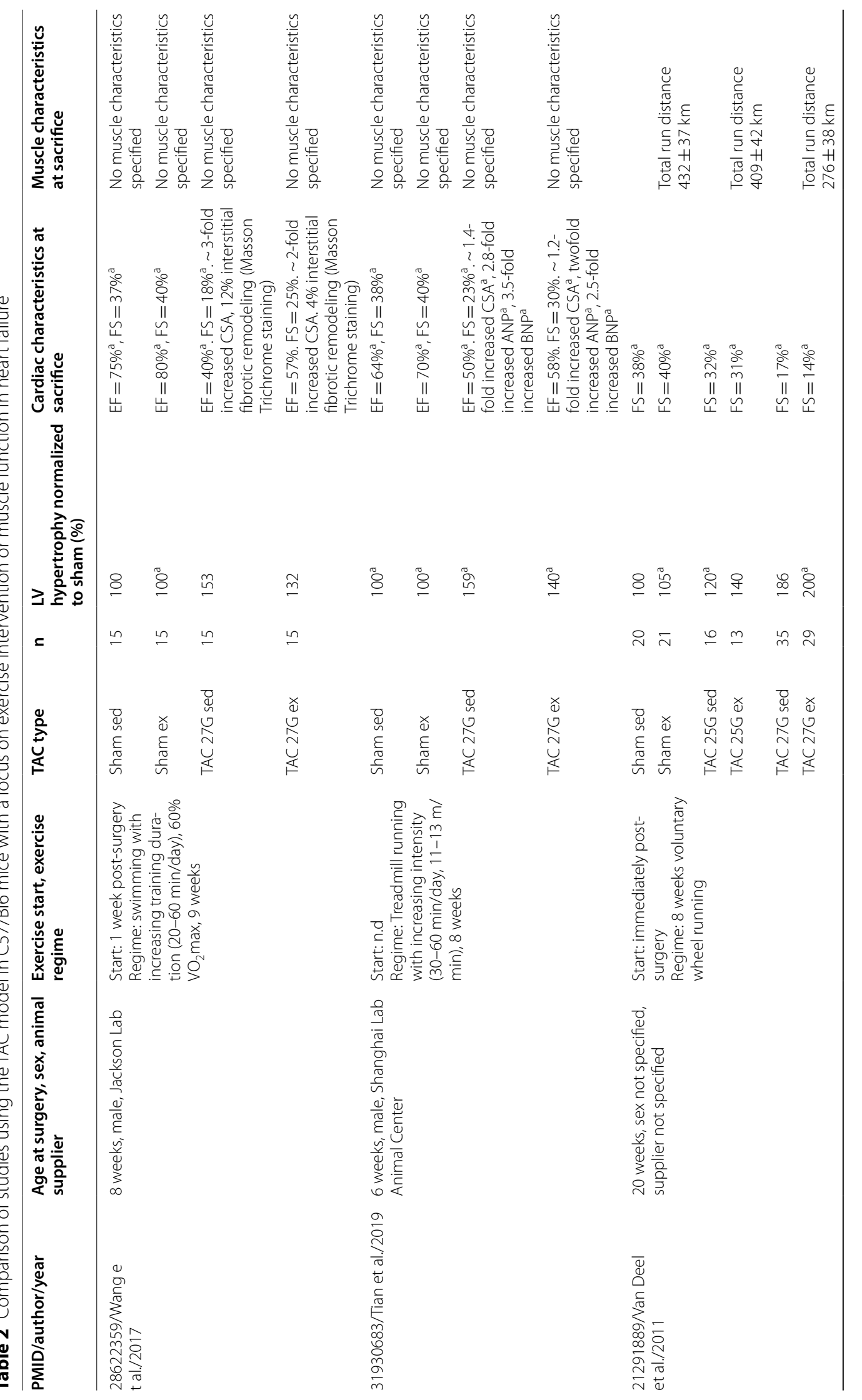




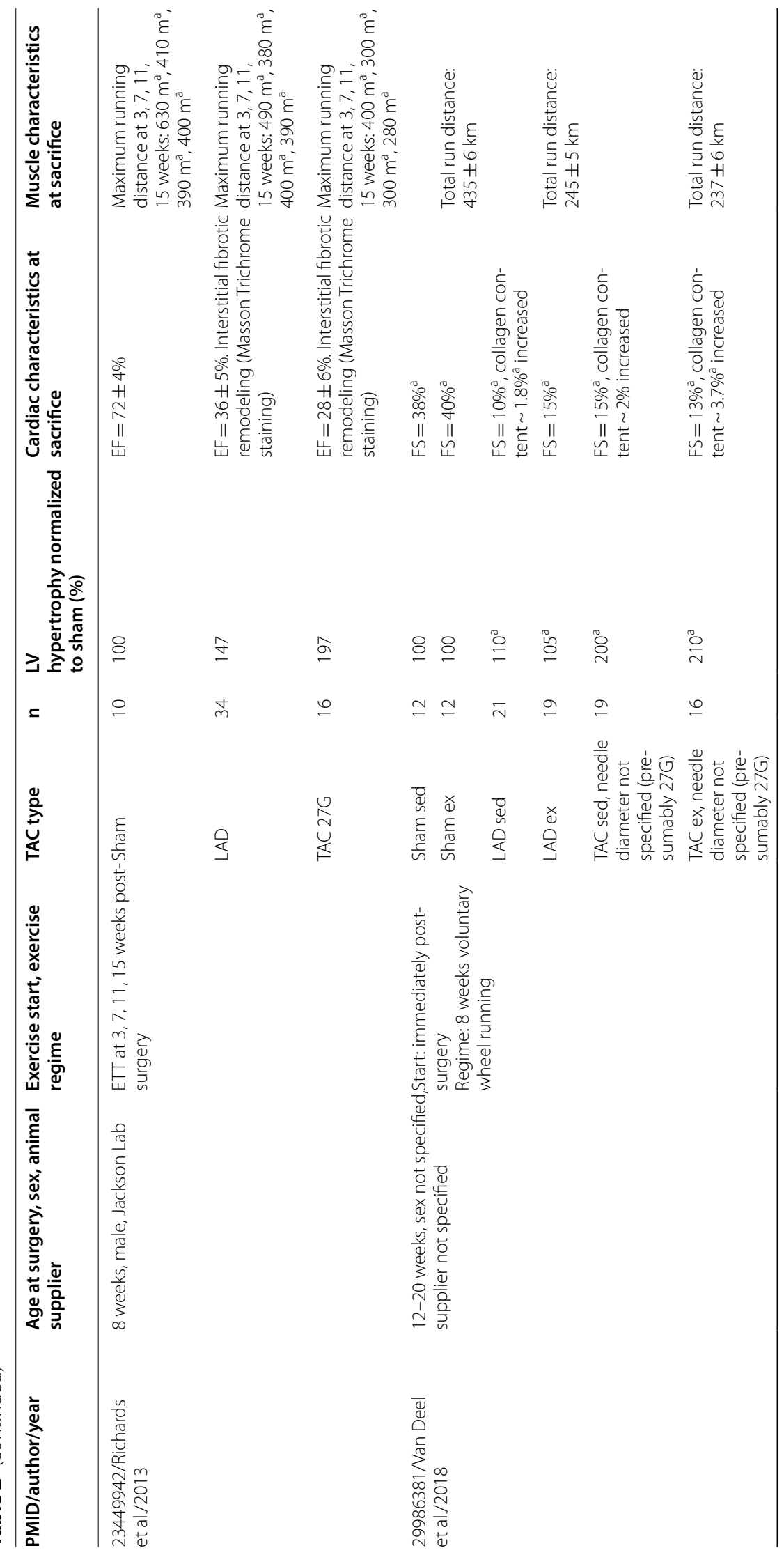




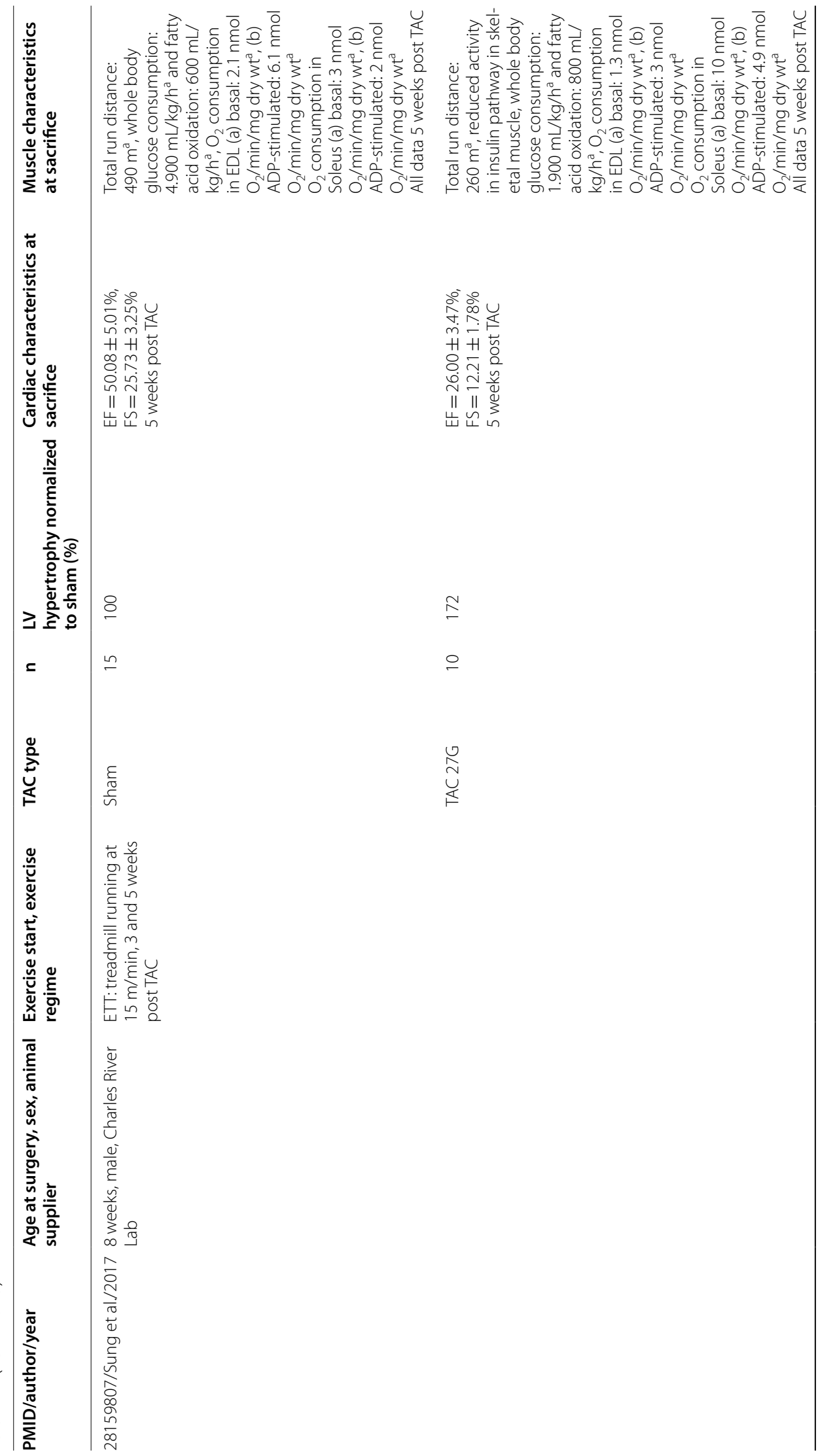




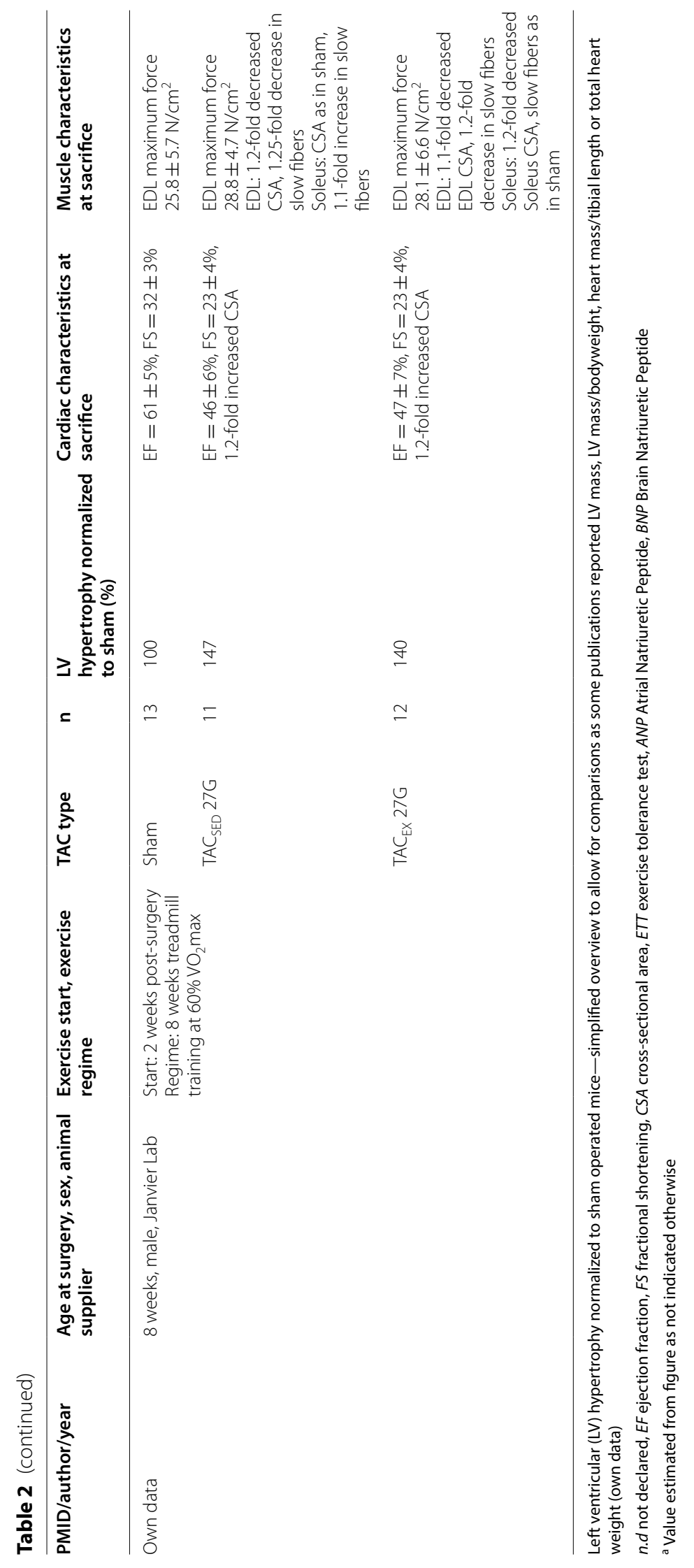


patients [6]. This is in strong contrast to the increasing intensity exercise regimes that were done by other groups [8, 18]. Van Deel et al. further observed an increase in LV mass in trained 25G TAC operated mice in contrast to sham. They showed a trending towards enhanced fibrosis following exercise training, accompanying the observed LV dysfunction [6], indicating that even voluntary exercise aggravates cardiac dysfunction in 25G mild TAC.

Richards as well as Gillis et al. characterized cardiac performance under moderate pressure overload applying 26G TAC $[16,33]$. Both found higher LV mass and increased cardiomyocyte CSA four and up to 18 weeks post TAC [16], overall indicating a deterioration of cardiac performance in the course of time. Richards et al. performed exercise tolerance test at 3, 7, 11 and 15 weeks post 27G TAC and detected increasing exercise intolerance accompanied by decreasing EF in a time dependent aggravation [34]. Sung et al. observed decreased treadmill running distances 3 and 5 weeks post $27 \mathrm{G}$ severe TAC indicating exercise intolerance in HF mice [35]. They observed deteriorated LV mass, EF and FS [35]. Additionally, van Deel et al. examined the effects of mild exercise training on 27G severe TAC, observing a further increased LV mass as well as decreased FS down to $14 \%$ [6]. This is in accordance to our data, as we observed further aggravated cardiac performance following exercise intervention $[6,7,34]$. Neither LV mass nor EF, FS or fibrosis development were improved by exercise intervention, but rather additionally aggravated. Interestingly, Wang and Tian found intense exercise intervention to have beneficial effects on 27G severe TAC [8, 18], although van Deel et al. found mild exercise in $25 \mathrm{G}$ mild TAC to already deteriorate cardiac function [6]. The underlying cause for these contrasting results remain unclear. One limitation to the studies by Wang and Tian is that they didn't test for maximum $\mathrm{VO}_{2}$ consumption before designing their exercise protocol $[8,18]$. Instead they increased exercise intensity and duration without adapting the protocol according to individual capability of the HF mice in the first place. Wang et al. declare no mice were floating during swimming exercise [8], but did not discuss fear-evoking, psychological effects [36] of swimming exercise to the animals. Tian et al. did not mention, if mice dropped out during treadmill running [18]. We think careful observation of the HF mice during exercise training is essential, because progressing HF results in increased mice dropouts due to exhaustion. This is in accordance with EDL contraction force of $\mathrm{TAC}_{\mathrm{EX}}$ showing no significant difference from Sham or $\mathrm{TAC}_{\mathrm{SED}}$. One reason for this observation may be the severely diminished perfusion of the skeletal muscle due to increased peripheral vasoconstriction in HF. This in turn is caused by the impaired cardiac output [37].

One pitfall in the studies of Richards [34] and van Deel [6] is the lack of histological or molecular analyses of skeletal muscle alterations of TAC operated mice with and without exercise training. Our data show no beneficial influence of moderate exercise training on skeletal muscle structure or function. To our knowledge, the study by Sung et al. is the only one to assess metabolic alterations in skeletal muscles 5 weeks post TAC. They found a reduced activity in the insulin pathway in skeletal muscles resulting in reduced whole body glucose consumption, increased fatty acid oxidation in EDL and soleus, but no alterations in force production of tibialis anterior and soleus muscle [35]. Especially the absence of HF-induced peripheral muscle strength loss [35] is in accordance to our study [6, 33, 34].

All other studies point out that increasing TAC severity is associated with worsening cardiac performance defining the HF phenotype. While in humans HF induces skeletal muscle atrophy [3], we could not observe this effect in mice. Additionally, we and others observed that moderate exercise further deteriorated cardiac function and promoted fiber atrophy in the TAC mouse model.

The data of the current study suggest that TAC in mice in combination with moderate exercise training is not suitable to ameliorate HF associated cardiac dysfunction.

Exercise itself leads to temporarily increased blood pressure, which physiologically can be regulated via peripheral impedance in healthy training individuals. In active muscles, resistance vessels relax to increase blood flow in order to meet the muscles metabolic requirements [38]. Outside the active muscle systemic vascular resistance decreases, which finally leads to an increased heart rate. The LV output is increased due to ensure proper nourishment of the active muscle [38]. In TAC operated mice systolic and diastolic LV filling pressure is significantly increased alongside with an increased vascular stiffness, vascular resistance as well as increased blood pressure and LV filling pressure [39]. These factors fix systemic vascular and LV load at a high level [39]. This condition restricts the capability to regulate blood pressure during exercise [6].

Finally, since physical activity is capable of correcting structural and metabolic alterations in the skeletal muscle of HF patients [4-8], the TAC mouse model especially in combination with exercise intervention does not mirror this. 


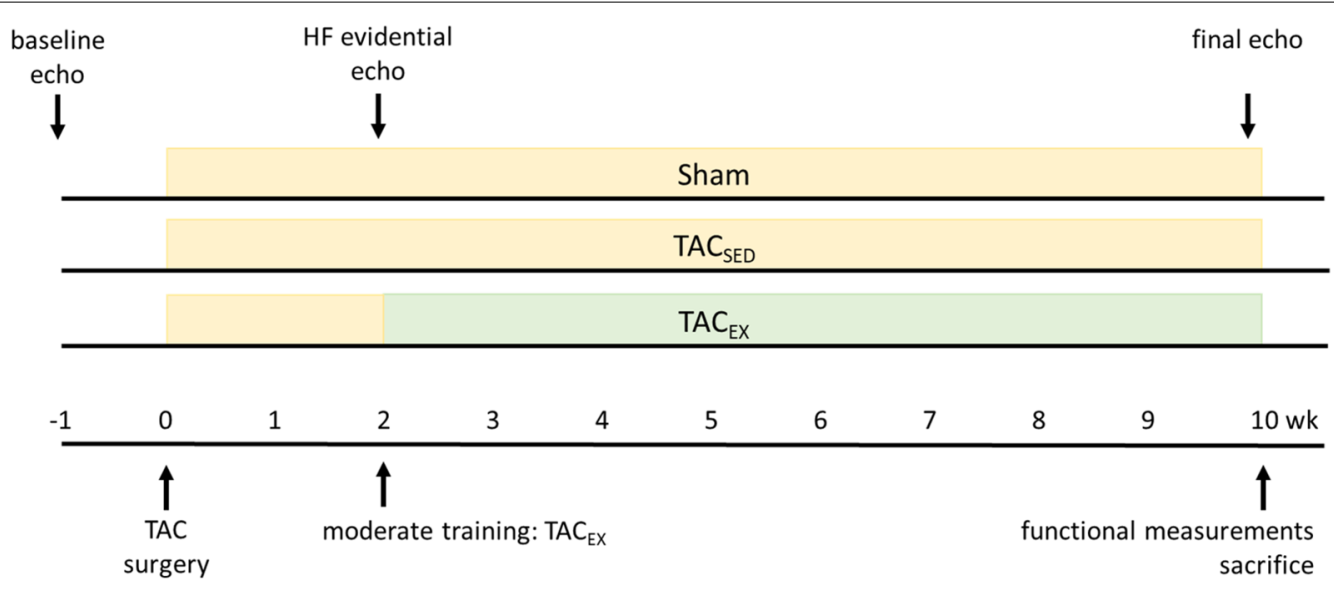

Fig. 4 Timeline of study course. $w k$ week

\section{Conclusion}

TAC is a solid model to study the effects of LV pressure overload in $\mathrm{C} 57 \mathrm{BL} / 6 \mathrm{~J}$ mice. Our data clearly indicate the suitability of the 27G TAC technique to introduce HF characteristics in mice. Cardiac and skeletal muscle remodeling and function is not ameliorated but rather enhanced by exercise intervention in the 27G TAC mouse model. Other TAC phenotypes (mild/25G or moderate/26G) may affect skeletal muscle structure and performance to a different degree, but this remains unclear.

We observed that TAC alters RV structure in addition to LV remodeling, which indicates development of a biventricular dysfunction. Thus, TAC in mice may be considered as an appropriate model for type $2 \mathrm{PH}$.

In summary, moderate exercise training intervention in the 27G TAC mouse model is not suitable to study beneficial exercise effects on cardiac remodeling and peripheral cachexia in HF.

\section{Materials and methods}

\section{Animals, TAC surgery, and sacrifice}

All experiments in this study were approved by the local Animal Research Authority, in accordance with the European Directive (TVV 51/18). A total of 45 male C57BL/6J mice (Janvier Labs, Le Genest-SaintIsle, France) entered the study (see Fig. 4 for study course). The 6 weeks old mice were randomized into two groups: sham-operated control $(n=15)$ and TACoperated group $(\mathrm{n}=30)$. A second randomization of the TAC-operated group was done 2 weeks after TAC surgery: TAC + exercise $(\mathrm{n}=15), \quad$ TAC + sedentary $(\mathrm{n}=15)$.
Eight-week old mice weighing 19-24 g, were subcutaneously administered with $5.0 \mathrm{mg} / \mathrm{kg}$ carprofene for preoperative analgesia. The mice were sedated by intraperitoneal injection of $0.5 \mathrm{mg} / \mathrm{kg}$ Medetomidin, $5 \mathrm{mg} /$ $\mathrm{kg}$ Midazolam und $0.05 \mathrm{mg} / \mathrm{kg}$ Fentanyl. The chest was opened through a lateral thoracic window from the throat to the second rip. A 27G needle was placed on the transverse aorta and secured using 6.0 prolene to reduce aortic diameter to $0.4 \mathrm{~mm}$. The needle was removed immediately and the chest was closed using 6.0 prolene. The sham-operated control group underwent the same procedure but without binding of the aorta. Post-operative analgesia comprised of $0.1 \mathrm{mg} /$ $\mathrm{kb}$ buprenorphine $3 \times$ per day, $5.0 \mathrm{mg} / \mathrm{kg}$ carprofene $1 \times$ per day and additional buprenorphine supply in the drinking water for 2-3 days in total. Mice were weighted and sacrificed 1 day after the last training unit using intraperitoneal injection of $5 \mathrm{mg} / \mathrm{kg}$ xylazinhydrochloride, $100 \mathrm{mg} / \mathrm{kg}$ ketaminhydrochloride and $0.1 \mathrm{mg} / \mathrm{kg}$ atropinsulfate. The hearts were separated, weighted and immediately stored in liquid nitrogen whereas the medial portion of the heart was fixed in $4 \%$ paraformaldehyde. Left extensor digitorum longus was removed after ligating it's proximal tendon using silk braid black, followed by dissecting the muscle without pressure or dragging and finally binding the distal EDL tendon for muscle functional measurement. Muscle preparation was conducted in carbogen $\left(95 \% \mathrm{O}_{2}\right.$, $5 \% \mathrm{CO}_{2}$ )-flushed Krebs-Henseleit-Buffer (120.5 mM $\mathrm{NaCl}, 4.8 \mathrm{mM} \mathrm{KCl}, 1.2 \mathrm{mM} \mathrm{MgSO}_{4}, 1.2 \mathrm{mM} \mathrm{NaH}_{2} \mathrm{PO}_{4}$, $20.4 \mathrm{mM} \mathrm{NaHCO}, 1.6 \mathrm{mM} \mathrm{CaCl}_{2}, 10 \mathrm{mM}$ dextrose, $1 \mathrm{mM}$ pyruvate, $\mathrm{pH}=7.4$ ). 
Table 3 Daily exercise regime of the moderate training group $T A C_{E X}$

\begin{tabular}{ll}
\hline & $\begin{array}{l}\text { Speed } \\
(\mathbf{m} / \\
\mathbf{m i n})\end{array}$ \\
\hline Warm up/40\% & 10 \\
Training/60\% & 16 \\
Cool down/40\% & 10 \\
\hline
\end{tabular}

\section{Echocardiographic measurement of cardiac function in the developing $\mathrm{HF}$}

For echocardiography (Vevo 770, Visual Sonics, Canada) mice were anesthetized with 5 vol\% isoflurane followed by a 2 vol\% isoflurane flow for maintaining anesthesia under continuous ventilation. Baseline measurements were performed at 7 weeks old mice ( $t=-1$ week) and again at an age of 10 weeks $(\mathrm{t}=2$ weeks $)$ and 18 weeks $(\mathrm{t}=10$ weeks $)$. Systolic and diastolic LV thickness, volume, ejection fraction and fractional shortening in control, HF and HF training groups were measured at the indicated time points (see Fig. 4).

\section{Exercise protocol}

The $\mathrm{TAC}_{\mathrm{EX}}$ group started exercise intervention 2 weeks after TAC operation five times a week for a total of 8 weeks. Maximum $\mathrm{VO}_{2}$ max was determined using an exercise tolerance test and a moderate training regime was defined at $60 \%$. According to the previously performed exercise tolerance test to estimate $\mathrm{VO}_{2} \max$, a training schedule was applied (see Table 3). Treadmill speed was adapted to the group's slowest individuum regarding the exercise tolerance test. Moderate training was performed in a treadmill at an inclination of $20^{\circ}$ including an initial $10 \mathrm{~min}$ warm up and final cool down at $40 \%$ of the maximum performance and a 40 min training phase at $60 \%$ of the maximum performance in between.

\section{Histological evaluation of TAC-induced hypertrophy and fibrosis}

Cardiac hypertrophy was evaluated by analyzing crosssections $(3 \mu \mathrm{m})$ were stained with hematoxylin and eosin. Area, circumference, as well as the thickness of the left and right ventricular wall were determined using ImageJ (v1.8, NIH, USA). Data are shown in relation to sham. Sirius Red staining was applied for identification and quantification of collagenous fibrotic tissue. For analysis of heart metrics ObjectJ PlugIn (Vischer, N., The Netherlands) was used to measure each ventricle wall 5 times at 5 positions and a polygonal region drawn around the cross section to calculate the heart area and circumference. For fibrosis estimation a threshold for Sirius Red staining intensity was applied and the proportion of interstitial and perivascular fibrosis was calculated in relation to the area of the whole section.

\section{Muscle function}

Left EDL muscle function was determined as described [40]. In brief, EDL was fixed on a force transducer (Aurora Scientific, Aurora, Canada) and equilibrated in an organ bath (Aurora Scientific, Aurora, Canada) at $37^{\circ} \mathrm{C}$ in carbogen-flushed Krebs-Henseleit-Buffer for $15 \mathrm{~min}$. To measure EDL force production the muscle was stimulated by an impulse of $700 \mathrm{~mA}$ and $0.25 \mathrm{~ms}$ with increasing frequencies $1,15,30,50,80,120,150$, $300 \mathrm{~Hz}$ followed by a one minute rest period using a bipolar high performance stimulator (Aurora Scientific, Aurora, Canada). The tension generated by the EDL muscle contraction is recorded in gram (g) as a function of frequency.

\section{Slow-fast muscle fiber staining}

Serial formalin-fixed and paraffin-embedded EDL and Soleus slices $(3 \mu \mathrm{m})$ were stained for histological analyses of cross-sectional area. First muscle tissue slices were deparaffinized in $3 \times 10 \mathrm{~min}$ xylol followed by a descending ethanol series hydration. Antigen retrieval was conducted in target retrieval solution (Dako, Santa Clara, USA). After cooling to room temperature, the slices were washed with deionized water and treated with $(20 \mu \mathrm{g} / \mathrm{mL})$ Proteinase K (Sigma Aldrich, St. Louis, USA) for $10 \mathrm{~min}$ at $37{ }^{\circ} \mathrm{C}$, washed with $1 \times$ PBS and treated with Peroxidase Block (Dako, Santa Clara, USA) for $10 \mathrm{~min}$ at room temperature, washed again with $1 \times$ PBS and blocked with serum-free Protein Block (Dako, Santa Clara, USA) for $20 \mathrm{~min}$ at room temperature. After another washing step in $1 \times$ PBS slices were incubated with an anti-slow skeletal MHC antibody (Abcam, Cambridge, UK) at $4{ }^{\circ} \mathrm{C}$ overnight. Slices were washed again in $1 \times$ PBS and incubated with a peroxidase-conjugated secondary antibody (Sigma Aldrich, St. Louis, USA) at room temperature for $45 \mathrm{~min}$, washed again with $1 \times \mathrm{PBS}$ and processed using VECTASTAIN Elite ABC Kit Peroxidase and the DAB Peroxidase Substrate Kit (Vector laboratories, San Diego, USA).

\section{Data processing and statistics}

Heart weight was normalized to body weight, or to tibial length to compensate potential differences in BW. 
Statistical analyses were performed using GraphPadPrism6 (San Diego, CA, USA). Data are presented as mean \pm standard error of the mean (SEM). Shapiro-Wilks-Test was performed to check for normal distribution. Group differences were analyzed using one-way ANOVA or Dunnett's multiple comparison. Muscle function (force-time-relationship) was assessed by two-way repeated measures ANOVA. Bonferroni correction was used as posttest. All $\mathrm{p}$-values $<0.05$ were considered statistically significant.

\section{Abbreviations}

BW: Body weight; CSA: Cross-sectional area; EDL: Extensor digitorum longus; EF: Ejection fraction; ETT: Exercise tolerance test; FS: Fractional shortening; G: Gauge; HF: Heart failure; LCCA: Left common carotid artery; LV: Left ventricle/left ventricular; LVEF: Left ventricular ejection fraction; $\mathrm{PH}$ : Pulmonary hypertension; RV: Right ventricle/right ventricular; TAC: Transverse aortic constriction; TAC $_{\mathrm{EX}}$ : Transverse aortic constriction combined with exercise; TAC $_{S E D}$ : Transverse aortic constriction combined in sedentary mice; TL: Tibial length.

\section{Acknowledgements}

We acknowledge support from Leipzig University for Open Access Publishing.

\section{Authors' contributions}

VA, HT, PB: conceptualization. SW, PB: sample acquisition, functional testing AK, SK: histological analyzes. PB, HT, VA, JB: analyzation, manuscript compilation. All authors contributed to the interpretation of the data. All authors critically revised the manuscript, are fully accountable for its content, and agreed with the submission of the manuscript. All authors read and approved the final manuscript.

\section{Funding}

Open Access funding enabled and organized by Projekt DEAL.

\section{Availability of data and materials}

Not applicable.

\section{Declarations}

Ethics approval and consent to participate

Not applicable.

\section{Consent for publication}

Not applicable.

\section{Competing interests}

The authors declare no competing interests.

\section{Author details}

${ }^{1}$ Department of Cardiology, Heart Center Leipzig at University of Leipzig, Strümpellstr. 39, 04289 Leipzig, Germany. ${ }^{2}$ Laboratory of Molecular and Experimental Cardiology, TU Dresden, Heart Center Dresden, Dresden, Germany.

\section{Received: 28 May 2021 Accepted: 30 Auqust 2021}

Published online: 19 September 2021

\section{References}

1. Groenewegen A, Rutten FH, Mosterd A, Hoes AW. Epidemiology of heart failure. Eur J Heart Fail. 2020;22:1342-56.
2. Jones NR, Roalfe AK, Adoki I, Hobbs FDR, Taylor CJ. Survival of patients with chronic heart failure in the community: a systematic review and meta-analysis. Eur J Heart Fail. 2019;21:1306-25.

3. Filippatos GS, Anker SD, Kremastinos DT. Pathophysiology of peripheral muscle wasting in cardiac cachexia. Curr Opin Clin Nutr Metab Care. 2005;8:249-54

4. Zizola C, Schulze PC. Metabolic and structural impairment of skeletal muscle in heart failure. Heart Fail Rev. 2013;18:623-30.

5. Platt $\mathrm{C}$, Houstis N, Rosenzweig A. Using exercise to measure and modify cardiac function. Cell Metab. 2015;21:227-36.

6. van Deel ED, et al. Exercise training does not improve cardiac function in compensated or decompensated left ventricular hypertrophy induced by aortic stenosis. J Mol Cell Cardiol. 2011;50:1017-25.

7. Van Deel ED, Octavia Y, De Waard MC, De Boer M, Duncker DJ. Exercise training has contrasting effects in myocardial infarction and pressure overload due to divergent endothelial nitric oxide synthase regulation. Int J Mol Sci. 2018;19:1968.

8. Wang $B$, et al. Aerobic exercise protects against pressure overloadinduced cardiac dysfunction and hypertrophy via beta3-AR-nNOS-NO activation. PLOS ONE. 2017:12:e0179648.

9. Hansen D, Dendale P, van Loon LJ, Meeusen R. The impact of training modalities on the clinical benefits of exercise intervention in patients with cardiovascular disease risk or type 2 diabetes mellitus. Sports Med. 2010;40:921-40.

10. Benito B, Nattel S. Exercise training as a treatment for heart failure: potential mechanisms and clinical implications. J Physiol. 2009:587:5011-3.

11. Pina $I L$, et al. Exercise and heart failure: a statement from the American Heart Association Committee on exercise, rehabilitation, and prevention. Circulation. 2003;107:1210-25.

12. Heran BS, et al. Exercise-based cardiac rehabilitation for coronary heart disease. Cochrane Database Syst Rev. 2011. https://doi.org/10.1002/ 14651858.CD001800.pub2.

13. Duncker DJ, et al. Exercise training in adverse cardiac remodeling Pflugers Arch. 2014;466:1079-91.

14. Reichert K, et al. Murine left anterior descending (LAD) coronary artery ligation: an improved and simplified model for myocardial infarction. J Vis Exp. 2017;2(122):e55353.

15. deAlmeida AC, van Oort RJ, Wehrens XH. Transverse aortic constriction in mice. J Vis Exp. 2010;38:e1729.

16. Richards DA, et al. Distinct phenotypes induced by three degrees of transverse aortic constriction in mice. Sci Rep. 2019:9:5844.

17. Maillet M, van Berlo JH, Molkentin JD. Molecular basis of physiological heart growth: fundamental concepts and new players. Nat Rev Mol Cell Biol. 2013;14:38-48.

18. Tian $\mathrm{X}$, et al. Heat shock transcription factor 1 regulates exerciseinduced myocardial angiogenesis after pressure overload via HIF1alpha/NEGF pathway. J Cell Mol Med. 2020;24:2178-88.

19. Yang $F$, et al. Coronary artery remodeling in a model of left ventricular pressure overload is influenced by platelets and inflammatory cells. PLoS ONE. 2012;7:e40196.

20. Barrick CJ, Rojas M, Schoonhoven R, Smyth SS, Threadgill DW. Cardiac response to pressure overload in 129S1/SvImJ and C57BL/6J mice: temporal- and background-dependent development of concentric left ventricular hypertrophy. Am J Physiol Heart Circ Physiol. 2007;292:H2119-2130

21. van Nierop BJ, et al. Phenotyping of left and right ventricular function in mouse models of compensated hypertrophy and heart failure with cardiac MRI. PLoS ONE. 2013;8:e55424.

22. Platt MJ, Huber JS, Romanova N, Brunt KR, Simpson JA. Pathophysiological mapping of experimental heart failure: left and right ventricular remodeling in transverse aortic constriction is temporally, kinetically and structurally distinct. Front Physiol. 2018;9:472.

23. Vonk Noordegraaf A, Westerh of BE, Westerhof $\mathrm{N}$. The relationship between the right ventricle and its load in pulmonary hypertension. J Am Coll Cardiol. 2017:69:236-43.

24. Stenmark KR, Meyrick B, Galie N, Mooi WJ, McMurtry IF. Animal models of pulmonary arterial hypertension: the hope for etiological discovery 
and pharmacological cure. Am J Physiol Lung Cell Mol Physiol. 2009;297:L1013-1032.

25. Shah SJ. Pulmonary hypertension. JAMA. 2012;308:1366-74.

26. Pahal P, Sharma S. Secondary pulmonary hypertension. StatPearls. 2020

27. Farah C, Kleindienst A, Bolea G, Meyer G, Gayrard S, Geny B, Obert P, Cazorla O, Tanguy S, Reboul C. Exercise-induced cardioprotection: a role for eNOS uncoupling and NO metabolites. Basic Res Cardiol. 2013;108(6):389.

28. Powers SK, Smuder AJ, Kavazis AN, Quindry JC. Mechanisms of exercise-induced cardioprotection. Physiology. 2014;29:27-38.

29. Mann N, Rosenzweig A. Can exercise teach us how to treat heart disease? Circulation. 2012;126:2625-35.

30. Laughlin MH, et al. Peripheral circulation. Compr Physiol. 2012;2:321-447.

31. Balligand JL, Feron O, Dessy C. eNOS activation by physical forces: from short-term regulation of contraction to chronic remodeling of cardiovascular tissues. Physiol Rev. 2009;89:481-534.

32. Golbidi S, Laher I. Molecular mechanisms in exercise-induced cardioprotection. Cardiol Res Pract. 2011:2011:972807.

33. Gillis TE, et al. Dissecting the role of the myofilament in diaphragm dysfunction during the development of heart failure in mice. Am J Physiol Heart Circ Physiol. 2016;310:H572-586.
34. Richards DA, et al. Examining the relationship between exercise tolerance and isoproterenol-based cardiac reserve in murine models of heart failure. J Appl Physiol. 2013;1985(114):1202-10.

35. Sung MM, et al. Resveratrol improves exercise performance and skeletal muscle oxidative capacity in heart failure. Am J Physiol Heart Circ Physiol. 2017;312:H842-53.

36. Drugan RC, Christianson JP, Warner TA, Kent S. Resilience in shock and swim stress models of depression. Front Behav Neurosci. 2013;7:14.

37. Wilson JR, Martin JL, Schwartz D, Ferraro N. Exercise intolerance in patients with chronic heart failure: role of impaired nutritive flow to skeletal muscle. Circulation. 1984;69:1079-87.

38. Shepherd JT. Circulatory response to exercise in health. Circulation. 1987:76:VI3-10.

39. Lindman BR, Otto CM. Time to treat hypertension in patients with aortic stenosis. Circulation. 2013;128:1281-3.

40. Bowen TS, et al. Small-molecule inhibition of MuRF1 attenuates skeletal muscle atrophy and dysfunction in cardiac cachexia. J Cachexia Sarcopenia Muscle. 2017;8:939-53.

\section{Publisher's Note}

Springer Nature remains neutral with regard to jurisdictional claims in published maps and institutional affiliations.
Ready to submit your research? Choose BMC and benefit from:

- fast, convenient online submission

- thorough peer review by experienced researchers in your field

- rapid publication on acceptance

- support for research data, including large and complex data types

- gold Open Access which fosters wider collaboration and increased citations

- maximum visibility for your research: over 100M website views per year

At BMC, research is always in progress.

Learn more biomedcentral.com/submissions 